\title{
COI Regimen
}

National Cancer Institute

\section{Source}

National Cancer Institute. COI Regimen. NCI Thesaurus. Code C11899.

A chemotherapy regimen consisting of capecitabine, oxaliplatin, and irinotecan that may be used for the treatment of colorectal cancer. 